\title{
Gradients in the Brain: The Control of the Development of Form and Function in the Cerebral Cortex
}

\author{
Stephen N. Sansom and Frederick J. Livesey \\ Gurdon Institute and Department of Biochemistry, University of Cambridge, Tennis Court Road, \\ Cambridge, CB2 1QN \\ Correspondence: rick@gurdon.cam.ac.uk
}

In the developing brain, gradients are commonly used to divide neurogenic regions into distinct functional domains. In this article, we discuss the functions of morphogen and gene expression gradients in the assembly of the nervous system in the context of the development of the cerebral cortex. The cerebral cortex is a mammal-specific region of the forebrain that functions at the top of the neural hierarchy to process and interpret sensory information, plan and organize tasks, and to control motor functions. The mature cerebral cortex is a modular structure, consisting of anatomically and functionally distinct areas. Those areas of neurons are generated from a uniform neuroepithelial sheet by two forms of gradients: graded extracellular signals and a set of transcription factor gradients operating across the field of neocortical stem cells. Fgf signaling from the rostral pole of the cerebral cortex sets up gradients of expression of transcription factors by both activating and repressing gene expression. However, in contrast to the spinal cord and the early Drosophila embryo, these gradients are not subsequently resolved into molecularly distinct domains of gene expression. Instead, graded information in stem cells is translated into discrete, region-specific gene expression in the postmitotic neuronal progeny of the stem cells.

In the developing brain, gradients are commonly used to divide neurogenic regions into distinct functional domains. Examples of such gradients are the sonic hedgehog (Shh) gradient responsible for specifying dorsoventral fates in the spinal cord (Ericson et al. 1997), and the double inverted gradients of the transcription factors En1/2 and Pax2/5, induced by morphogens secreted from the isthmic organizer that pattern the midbrain and hindbrain (Sato et al. 2004). In this article, we discuss the functions of morphogen and gene expression gradients in the assembly of the nervous system in the context of the development of the cerebral cortex. The neocortex is the mammal-specific region of the forebrain that functions at the top of the neural hierarchy to process and interpret sensory information, plan and organize tasks, and to control motor functions. A key aspect of the functional anatomy of the cerebral cortex is that it is a modular structure and, as is discussed, those

Editors: James Briscoe, Peter Lawrence, and Jean-Paul Vincent

Additional Perspectives on Generation and Interpretation of Morphogen Gradients available at www.cshperspectives.org

Copyright (C) 2009 Cold Spring Harbor Laboratory Press; all rights reserved; doi: 10.1101/cshperspect.a002519

Cite this article as Cold Spring Harb Perspect Biol 2009;1:a002519 
modules of neurons are generated from a neuroepithelial sheet by two forms of gradients: graded extracellular signals and a set of transcription factor gradients operating across the field of neocortical stem cells.

\section{THE NEOCORTEX: A SIX-LAYERED SHEET OF NEURONS DIVIDED INTO FUNCTIONAL AREAS}

In humans, the neocortex accounts for nearly three-quarters of the brain volume and contains more than twenty billion neurons (Mountcastle 1998). The neocortex is composed of six layers of neurons, and those layers differ markedly in neuronal cell type composition, cell density, and connectivity. The neurons of the neocortex are characteristically arrayed into vertical groups that span the cortical layers. In humans, neocortical columns are approximately $2 \mathrm{~mm}$ tall, have a diameter of $0.5 \mathrm{~mm}$, and contain approximately 60,000 neurons (Rakic 2008). Neurons within columns are highly interconnected and share a common function. At a finer scale, neocortical columns can be subdivided into mini or microcolumns, which contain tens to hundreds of neurons, and have been proposed to comprise the basic unit of the neocortex (Mountcastle 1998; Jones 2000; Rakic 2008). At the simplest level of description, the deepest layers of cortical neurons (layers 5 and 6) contain projection neurons that connect areas of the cortex to one another or to subcortical structures. Layer 4 is the layer within which most extracortical inputs arrive, particularly from the sensory thalamus, whereas the superficial layers are composed mainly of local circuits that form reciprocal connections with the deep layers (Fig. 1A) (Thomson and Lamy 2007).

Groups of neocortical columns are organized into functionally distinct neocortical areas, such that the cortex is a patchwork of functional modules, including areas for motor control, vision, and hearing (Fig. 1B). With some notable exceptions, neocortical areas lack obvious anatomical boundaries, being distinguishable only by differences in cytoarchitecture, chemoarchitecture, connectivity, and gene expression. In the first accurate histological analysis, Brodmann divided the human neocortex into areas based on serial sectioning to produce a map of cortical areas (Brodmann 2006). Brodmann's maps have been largely confirmed by subsequent investigations, including studies involving electrophysical perturbation in live subjects and neurosurgical procedures on conscious patients under local anesthesia (Mountcastle 1998). The functional importance of neocortical areas is also known from the examination of subjects that have suffered loss or damage to particular areas-for example, damage to Broca's or Wernicke's areas in the left hemisphere result in an inability to process language and generate speech.

In all species, the neocortex contains three major areas along the rostrocaudal axis (Fig. 1B): the rostral motor cortex, the medial somatosensory cortex, and the caudal visual cortex; although the relative sizes of those areas differs markedly in different species. Although the broad organization of neocortical areas shows remarkable similarity in different mammals, the evolution of the neocortex is marked by a dramatic increase in cortical volume and in the total number of cortical areas. The neocortex of a mammal such as the shrew has a surface area a thousand-fold smaller than that of a human and has 10-fold fewer functional areas (Striedter 2005). Understanding the mechanisms that pattern the neocortex into the adult areas represents a major challenge and focus of modern developmental neurobiology.

\section{DEVELOPMENT OF THE NEOCORTEX- THE RADIAL UNIT HYPOTHESIS}

In mice, a model organism commonly used for research in this area, development of the neocortex begins at embryonic day 9.5 (E9.5) with the appearance of the cerebral vesicles from the dorsal surface of the rostral neural tube. Initially, the neocortical primordium is comprised of an apparently homogenous pool of neural stem cells. The first postmitotic neurons of the neocortex, the Cajal-Retzius 
A

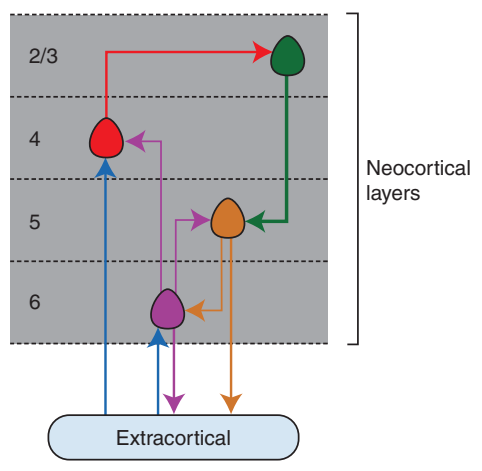

B

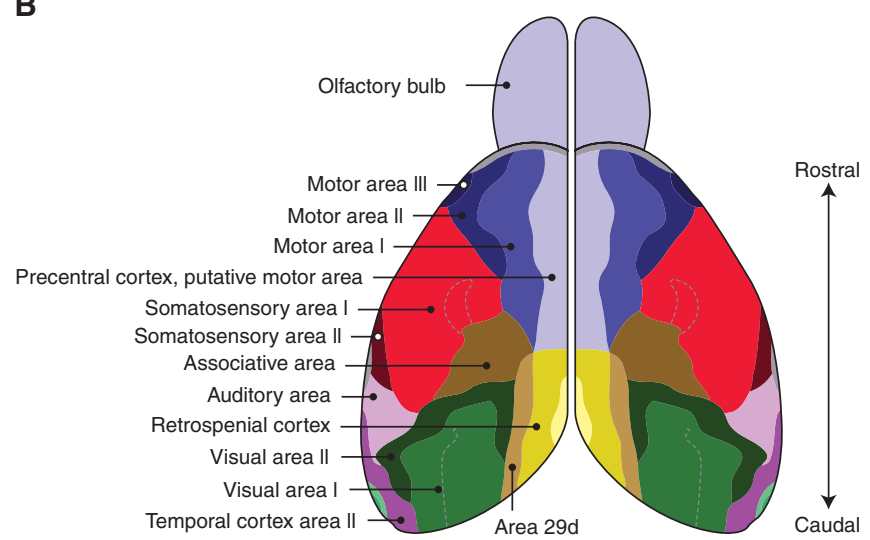

Figure 1. The arrangement of neocortical circuits and areas in the adult mouse brain. $(A)$ The basic cortical circuit. Major extracortical inputs terminate in layer 4 and to a lesser degree in layer 6. Layer 4 neurons project to layers 2 and 3, which in turn innervate layers 5 and 6, the major output layers of the cortex. $(B)$ Dorsal view of the adult mouse brain, with the functional roles of histologically defined areas labeled. Area maps redrawn from Wree et al. 1983.

cells, appear at E10.5 to form a transient structure known as the marginal zone that later becomes layer 1 . The subsequent generation of the glutamatergic projection neurons of layers $2-6$ by neocortical stem cells takes place from E11 until approximately E17, with neurons of deep layers (layer 6) produced before those of the outer layers (2/3) (Fig. 2A). Postmitotic layer neurons born in the VZ migrate radially outwards to form the cortical layers that together comprise the cortical plate. The outwards migration of neurons of layers VI to II takes place along the processes of radial glial cells that span the width of the developing neocortex (Fig. 2B). Neurons of layer 6 are first to leave the ventricular zone and migrate radially to form the nascent cortical plate. Neurons of layer V to II then migrate past those of layer VI and adopt successively superficial positions (Fig. 2A).

The radial migration of the neuronal progeny of neocortical stem cells suggested that they might remain in spatial register with their mother cell, and this important idea was proposed as the radial unit hypothesis (McConnell 1988; Rakic 1988b; Rakic 1990). Initial efforts to test the hypothesis, in which retroviral labeling was used to mark and visualize clones of neurons in the developing neocortex, failed to provide support for this model, instead finding evidence for both horizontal and radial clones of cells (Price and Thurlow 1988; Walsh and Cepko 1992; Walsh and Cepko 1993). However, after some debate, subsequent studies of the subcortical origins of inhibitory interneurons of the cortex and further lineage analyses confirmed the accuracy of the radial unit hypothesis (Tan and Breen 1993; Soriano et al. 1995; Anderson et al. 1997). These studies revealed that the glutamergic neuronal progeny of neocortical stem cells form radial columns that span the cortical plate (Tan and Breen 1993; Soriano et al. 1995) (Fig 2C). By contrast, the horizontal clones observed in the initial retroviral studies were found to represent the tangential dispersion of inwardly migrating inhibitory GABAergic interneurons arriving from the ganglionic eminences of the ventral forebrain (O'Rourke et al. 1995; Anderson et al. 1997; Wonders and Anderson 2006). Thus, the postmitotic neuronal progeny of neocortical stem cells migrate radially upward to form the layers and areas of the adult neocortex. Importantly, the radial unit hypothesis gave rise to the idea that a spatial pattern in 
S.N. Sansom and F.J. Livesey

A
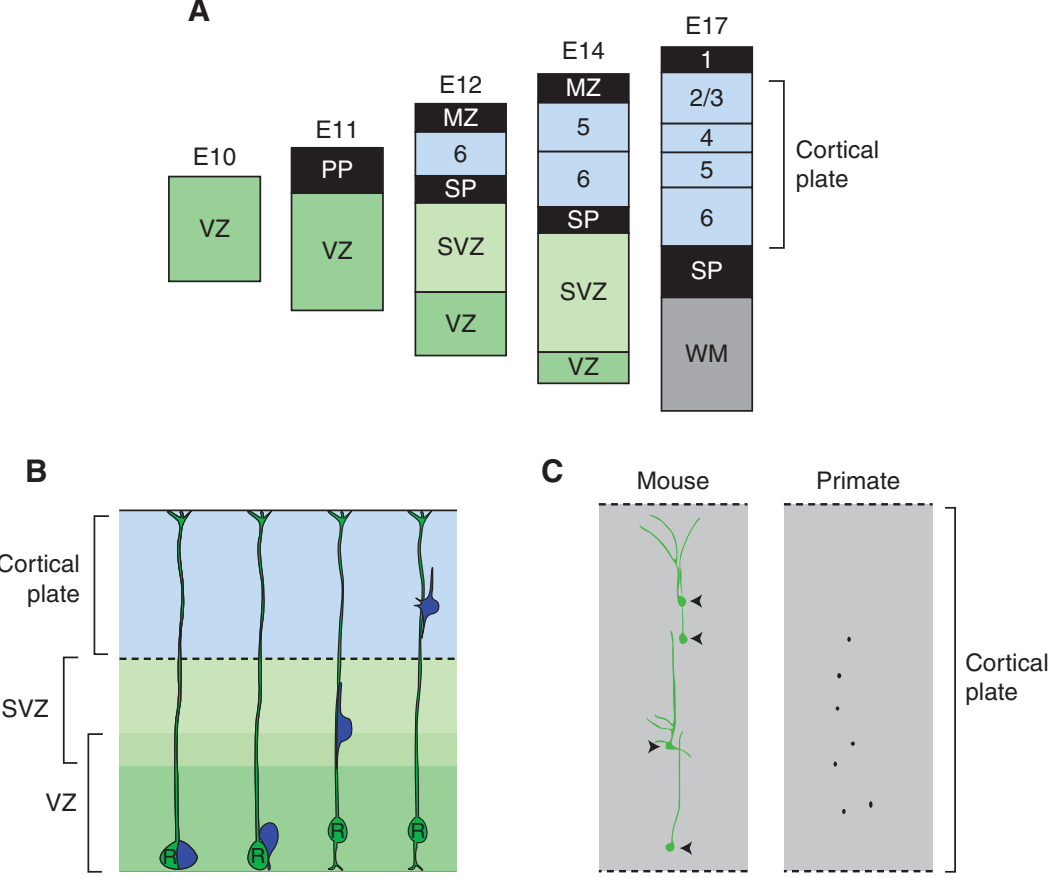

Figure 2. Cortical stem cells are multipotent, generating neurons for each layer in a fixed temporal order. (A) Layer-specific neurons are generated in a fixed temporal order in a classic inside-out pattern over 6 days in the mouse cortex. (B) Neurons (blue) and generated by radial glia stem cells (green) in the ventricular zone and subsequently migrate radially outwards into the cortical plate along the processes of the radial glia cells that span the width of the developing neocortex. $(C)$ Cortical stem cells generate radially arranged clones of neurons in mice and primates. Examples of retrovirally labeled clones are redrawn from Kornack and Rakic 1995 and Yu et al. 2009.

neocortical stem cells is transferred to the neurons of the cortical plate.

\section{NEOCORTICAL AREA FORMATION}

Areas of the neocortex cannot be distinguished by cytoarchitecture until postnatal day 2 (P2) in the mouse, although at birth several genes show region-specific expression in the cortical plate in partial correspondence to the emerging area boundaries. These include cell adhesion molecules (Cad6, Cad8), the orphan nuclear receptor RORß, the HLH transcription factor $\mathrm{Id} 2$, a neurotrophin receptor ( $\mathrm{p} 75)$, and molecules involved in cell migration and axon guidance, such as EphA7 and ephrin-A5 (Bishop et al. 2002). Remarkably, these genes show different rostrocaudal extents of expression in each layer of the neocortex. For example, although Id2 has a defined border of expression in layer-5 neurons that corresponds to the future boundary between somatosensory area 1 (S1) and motor area 1 (M1), in layers 2 and 3 , Id2 has a graded expression that continues across the entire tangential extent of the neocortex (Rubenstein et al. 1999; Garel et al. 2003). At present, there is little evidence for gene expression that is cleanly area-specific.

The question of neocortical area specification gave rise to two competing hypotheses: the protocortex hypothesis and the protomap hypothesis. In the protocortex hypothesis (van der Loos and Woolsey 1973; O'Leary 1989), it was suggested that area pattern in the developing neocortex was extrinsically specified by the innervating thalamocortical afferents (TCAs), sensory afferent neurons that project from distinct nuclei of the thalamus to specific cortical areas. These axons begin innervating the neocortex from E14.5, and by E15.5 have begun to 
extend through the cortical plate. In contrast, the protomap hypothesis proposed that neocortical areas were patterned from a map of spatial identity intrinsic to neocortical stem cells (Rakic 1988a). Thus, while the protocortex hypothesis advanced the idea that outside factors patterned a naïve cortical primordium or "tabula rasa," the protomap hypothesis argued that neocortical area pattern was intrinsically established by neocortical stem cells.

Several lines of evidence now show that the protomap hypothesis best describes the initial stages of neocortical patterning. First, heterotopic transplantation studies have shown that neocortical stem cells become regionally specified between E11 and E12, before the arrival of the thalamocortical afferents (Cohen-Tannoudji et al. 1994; Gitton et al. 1999a,b; Gaillard et al. 2003). Second, in both Gbx-2 and Mash 1 mutant mice, in which the TCA projection is respectively impaired or absent, region-specific gene expression is still observed in the cortical plate (Miyashita-Lin et al. 1999; Liu et al. 2000). Finally, additional support for the protomap hypothesis came from the identification of genes differentially expressed across the neocortical primordium before the arrival of the subcortical afferents. Notable among those genes are transcription factors expressed in opposing rostral-caudal gradients. For example, Pax6 is expressed in a rostrolateral high to caudomedial low gradient; Emx2 has a caudomedial high to rostrolateral low gradient; and COUP-TFI displays a caudolateral high to rostromedial low gradient (Walther and Gruss 1991; Simeone et al. 1992; Stoykova and Gruss 1994; Gulisano et al. 1996; Mallamaci et al. 1998; Liu et al. 2000). Other classes of genes commonly associated with development, such as the Ephs and Ephrins, and Cadherins, were also found to be expressed in gradients in neocortical stem cells (Mackarehtschian et al. 1999; Nakagawa et al. 1999). These gradients of gene expression provided the means by which a neocortical protomap might be encoded in neocortical stem cells.

However, although strong evidence supports the presence of an intrinsic pattern or protomap in the neocortical primordium, a role for TCAs in the differentiation, refinement, and maintenance of area identity has also been established (Frost and Schneider 1979; Sur et al. 1988; Schlaggar and O'Leary 1991; CohenTannoudji et al. 1994; Nothias et al. 1998; Gitton et al. 1999a,b). The protomap versus protocortex debate is therefore now resolved into a synthesis. Spacial information is conferred on neurons of the cortical plate from a protomap intrinsic to the neocortical primordium. This area pattern of the cortical plate is then refined and maintained by the innervating thalamocortical afferents. This model of neocortical area specification immediately raises two key questions: How is the neocortical protomap established and how is it interpreted in neocortical stem cells to give rise to regionspecific gene expression in the cortical plate?

\section{SECRETED GROWTH FACTORS PATTERN THE ROSTROCAUDAL AXIS OF THE NEOCORTEX}

The idea that secreted signaling factors might pattern a neocortical protomap has existed for decades (Creutzfeldt 1977; Rakic 1988a; Kuljis and Rakic 1990; Barbe and Levitt 1991; Rakic 1991). The first demonstration that such a patterning mechanism is used to generate the area map came from Fukuchi-Shimogori and Grove, who investigated the role of fibroblast growth factor (FGF) signaling in area specification (Fukuchi-Shimogori and Grove 2001). The expression of FGFs 3, 8, 17, 15, and 18 from E9.5 until E12.5 at the rostral midline of the neocortex in the commissural plate and surrounding tissue suggested the presence of a rostral, FGF-secreting signaling center (Bachler and Neubüser 2001).

In an elegant study, Fukuchi-Shimogori and Grove performed in vivo electroporation experiments to investigate the role of FGF8 in patterning the neocortex (Fukuchi-Shimogori and Grove 2001). Electroporations were performed at E11.5, at the time when the generation of the neuronal layers is just beginning. Augmenting the endogenous rostral FGF8 source led to an expansion of rostral cortical areas at the expense of caudal areas, as assayed 


\section{S.N. Sansom and F.J. Livesey}

by the expression of regional cortical plate markers at P0. Conversely, it was found that reduction of the rostral FGF signal by the electroporation of a soluble FGF receptor (sFGFR3) caused a dramatic shrinkage of rostral neocortical areas, and this phenotype was also later observed in FGF8 hypomorphic mice (Fig. 3A) (Garel et al. 2003). Most strikingly, Fukuchi-Shimogori and Grove (2001) found that the introduction of an ectopic FGF8 source in the caudal cortex resulted in a partial mirror duplication of the whisker barrel field of the more rostral somatosensory cortex (Fig. 3A).

In addition to demonstrating that the secreted factor FGF8 specifies a rostral identity
A

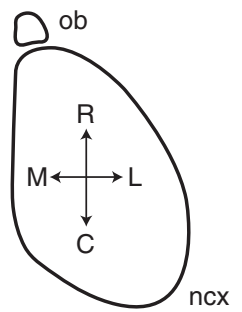

Wild type

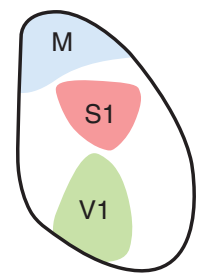

B
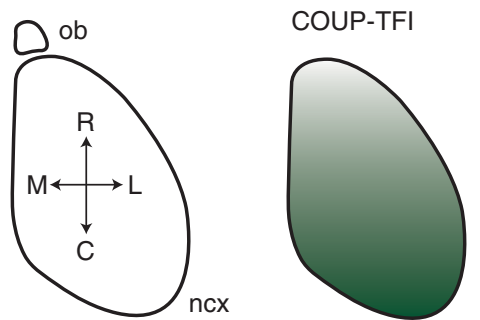

Wild type

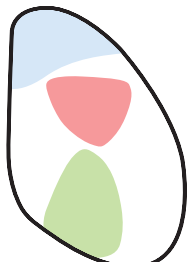

COUP-TFI CKO

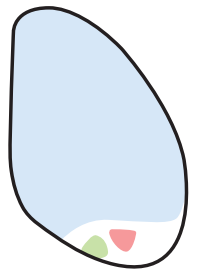

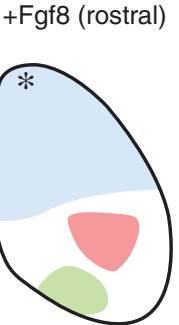

$\mathrm{Emx2}$

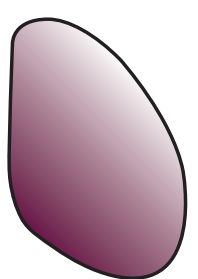

Emx2 null

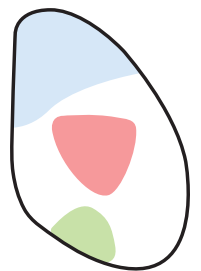

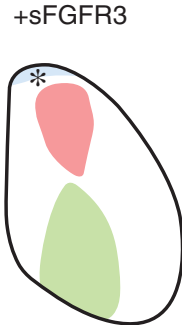

\section{+ Fgf8 (caudal)}
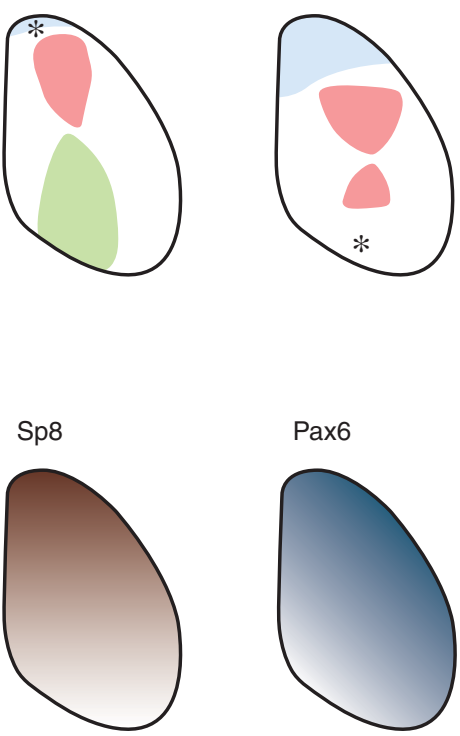

Sp8 CKO

Pax6 null
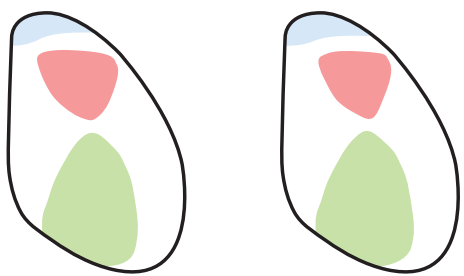

Figure 3. The roles of FGF signaling and graded transcription factor expression in neocortical pattern formation. (A) Diagram of a dorsal view of the mouse cortex (ncx, neocortex; ob, olfactory bulb; M, medial; L, lateral; $\mathrm{R}$, rostral; $\mathrm{C}$, caudal), with the major axes and areas labeled ( $\mathrm{M}$, motor; $\mathrm{S} 1$, somatosensory; V1, primary visual). The effects of altering the levels and positions of FGF8 signaling are shown. Increasing FGF8 levels rostrally increases the size of the rostral motor area at the expense of caudal areas. Conversely, antagonizing FGF8 signaling by expression of the extracellular face of an FGF receptor (sFGFR3) results in a reduction of the size of M1 and an increase in the size of caudal areas. Introduction of a new, caudal source of FGF8 results in the generation of a mirror-image of the $\mathrm{S} 1$ area in caudal cortex. $(B)$ The transcription factors COUP-TF1, Emx2, Sp8, and Pax6 are expressed in gradients along the rostrocaudal axis of the cortex as shown. The effects of null mutations in each transcription factor are shown (CKO, cortex-specific knockout). See text for details of each phenotype. 
in the neocortex, these studies suggested that it might function in the manner of a morphogen. In the classical model of a morphogen gradient, the morphogen is released from a localized source and acts in a concentration-dependent manner to specify two or more cell fates. In the neocortex, FGFs are released from a localized source at the rostral midline and the predictable shifts in regional gene expression on the reduction or augmentation of this source suggest that FGFs may act in a concentrationdependent manner to specify rostrocaudal positional identity in neocortical stem cells. Specifically, these data suggest that a high concentration of FGF specifies the rostral-most motor cortex, whereas lower concentrations direct somatosensory and visual cortical fates.

However, an FGF protein gradient across the neocortical ventricular zone has not been directly visualized, and the question of how such a gradient could form across the rostrocaudal axis of the neocortex remains unanswered. At the beginning of neocortical development (E9.5), the neocortical primordium is $300 \mu \mathrm{m}$ in length, approximately the same length as the syncytial blastoderm of the fruit fly, but then doubles in length within a day and reaches a length of $7000 \mu \mathrm{m}$ in the adult mouse (Loncar and Singer 1995; Lein et al. 2007). FGFs are produced by stem cells at the rostral midline and at the rostral pole of each cerebral hemisphere. One possibility is that FGFs diffuse solely through the pseudostratified neuroepithelium. Alternatively, FGFs may accumulate in the fluid-filled ventricles of both cerebral hemispheres, which are lined by the apical surfaces of the cortical stem cells. Therefore, it may be that an FGF gradient forms within this cavity across the ventricular surface of the developing neocortex.

Although definitive evidence for a gradient of FGF8 protein is lacking, evidence exists for a gradient of FGF signaling in the ventricular zone along the rostrocaudal axis. FGFs bind to and signal through four classical tyrosine kinase receptors (FGFR1-4), and three of these receptors are known to be expressed by neocortical stem cells. FGF receptors FGFR1, -2 , and -3 are all expressed in neocortical stem cells, with FGFR1 showing a high rostral to low caudal expression gradient, whereas FGFR2 and FGFR3 have low rostral to high caudal gradient of expression (Hébert et al. 2003). In mice, the forebrain-specific loss of FGFR1 causes a rostral shift in area identity, in addition to causing the loss of the olfactory bulbs, further supporting the idea that FGF functions as a rostrally secreted morphogen to specify area identity (Hébert et al. 2003).

Downstream of the receptors, the activation of the intracellular components of the FGF signaling pathway, such as the MAP kinases Erk1/2, has not been investigated in the cortex. However, the FGF inhibitors Sprouty1, -2, and -4 , which are members of the FGF synexpression group and direct targets of Ras-Erk signaling, are expressed in rostral-high gradients in the neocortex from E9.5 to E12.5, suggesting the presence of a gradient of active MAP kinase signaling in this tissue (Minowada et al. 1999; Mason et al. 2006). Furthermore, the expression gradients of Sprouty1 and -2 are diminished by reduced levels of FGF signaling (Cholfin and Rubenstein 2008).

The three members of the PEA3 group of the Ets domain transcription factors Erm (Etv5), Pea3 (Etv4), and Er81 (Etv1), are members of the FGF synexpession group and known transcriptional targets of FGF signaling (Buchwalter et al. 2004; de Launoit et al. 2006). In the developing neocortex, these transcription factors are expressed in nested rostralhigh to caudal-low gradients and the expression of these factors can be decreased or increased by reducing or augmenting the rostral FGF source (Fukuchi-Shimogori and Grove 2003). Finally, two other genes, the transcription factor Sp8 and the imprinted gene Mest/Peg1, the expression of which is induced by FGF signaling in the neocortex, are also expressed in rostralhigh to caudal gradient across the neocortical primordium (Sansom et al. 2005; Sahara et al. 2007; Zembrzycki et al. 2007).

Thus, FGFs pattern the cortex to form neocortical areas. Although it remains formally possible that FGF signaling acts indirectly or through secondary signals to do so, strong evidence at many levels of the FGF signaling 
S.N. Sansom and F.J. Livesey

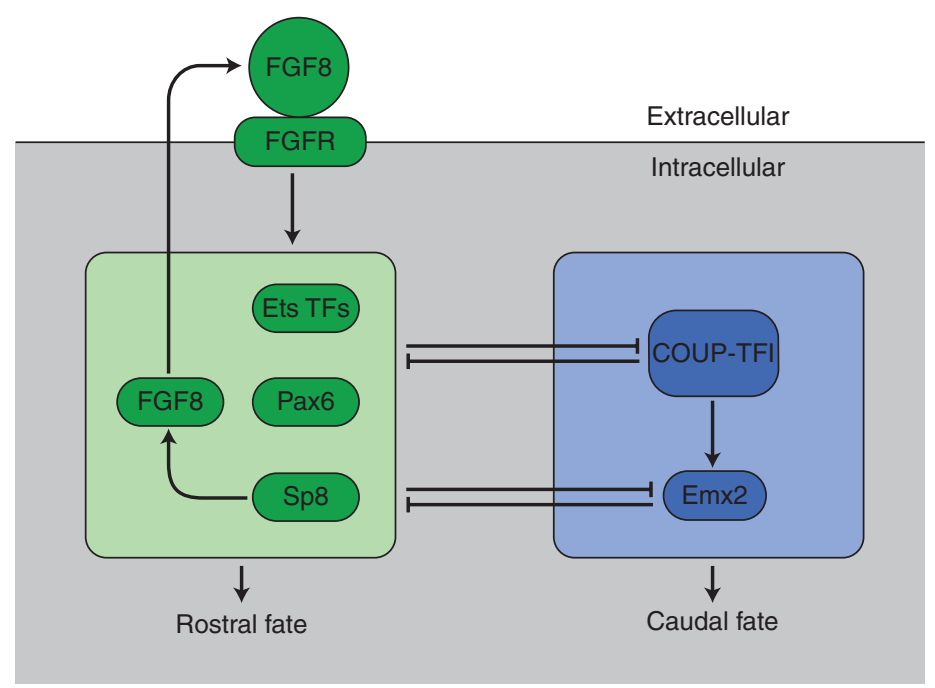

Figure 4. The outline of a cellular network for controlling neocortical area formation. FGF8, signaling through FGF receptors, induces or increases expression of the rostrally expressed transcription factors, the ETS factors Pax6 and FGF8. Sp8 in turn increases expression of Fgf8 rostrally. The rostral and caudal transcription factors show a degree of mutual cross-repression. Among the caudal transcription factors, COUP-TFI appears to be upstream of Emx2, as loss of Emx2 does not alter COUP-TFI expression, and COUP-TFI-null cortices have a more severe patterning phenotype than that observed in Emx2 nulls.

pathway suggests that FGFs directly pattern the cortex in a concentration-dependent manner. FGF signaling acts to determine positional identity in the neocortex by controlling a cellular network that includes several interacting transcription factors, the elements of which are considered below and summarized in Figure 4.

\section{FGF SIGNALING ESTABLISHES AN OPPOSING GRADIENT OF THE CAUDALISING TRANSCRIPTION FACTOR COUP-TFI}

The orphan nuclear receptor COUP-TFI is a transcription factor expressed in a caudomedialhigh to rostrolateral-low gradient in the developing neocortex (Zhou et al. 2001) (Fig. 3B). The roles of COUP-TFI in cortical development have been studied in germline and cortexspecific mutant mice, and have also been investigated by in vivo overexpression (Zhou et al. 2001; Armentano et al. 2007; Faedo et al. 2008). In mice with a cortex-specific deletion of COUP-TFI, the expression of regional cortical plate markers Cdh8, Id2, Fezf2, and Efna5 revealed that the balance of area pattern was changed such that the majority of the cortex was transformed into motor cortex, and the somatosensory and visual areas were greatly reduced in size and located at the caudal pole of the structure (Fig. 3B) (Armentano et al. 2007). COUP-TFI is therefore crucial for promoting a caudal identity in the neocortex. The rostralization of the neocortex in the absence of COUP-TFI, and the established role for COUP-TFI as a transcriptional repressor, suggest that COUP-TFI acts to specify caudal neocortical identity by repressing a rostral fate.

The gradient of COUP-TFI in the developing neocortex lies in opposition to the rostralhigh to caudal-low gradient of FGF signaling, and it is natural to ask whether these gradients regulate one another. There is good evidence that FGF signaling regulates COUP-TFI expression. The sensitivity of the COUP-TFI gradient to levels of FGF signaling has been shown both in vivo and in vitro. Augmentation of the rostral FGF source via in 
utero electroporation results in a contraction of the COUP-TFI gradient, whereas reduction of the endogenous FGF source induces a rostral expansion of COUP-TFI expression (FukuchiShimogori and Grove 2003; Garel et al. 2003). In vitro experiments have shown that COUPTFI is rapidly down-regulated in cortical explants in response to elevated FGF levels (Sansom et al. 2005). Interestingly, binding sites for the FGF target genes, the Ets transcription factors, have been identified in the COUP-TFI promoter, suggesting a possible mechanism for the regulation of COUP-TFI by FGF signaling (Salas et al. 2002). The regulation of FGF signaling by COUP-TFI is less well understood. However, reduced levels of phospho-Erk1/2 have been reported in the neocortex of mice constitutively overexpressing COUP-TFI, indicating that COUP-TFI can negatively regulate FGF signaling (Faedo et al. 2008).

The ability of FGF to repress the expression of COUP-TFI indicates that the Fgf-signaling gradient is responsible for establishing the caudal-high to rostral-low gradient of COUPTFI in the neocortex, in a manner analogous to that of the repression of dorsally expressed class II transcription factors in the spinal cord by the ventrally secreted morphogen sonic hedgehog (Briscoe and Ericson 2001). This suggests a default caudal identity for neocortical stem cells in the absence of Fgf signaling, and in support of this supposition, neocortical stem cells produced by directed differentiation from embryonic stem cells express COUP-TFI (Gaspard et al. 2008).

\section{GRADIENTS OF TRANSCRIPTION FACTORS REFINE AREA PATTERNING}

Downstream of FGF signaling and COUP-TFI, gradients of transcription factor expression across the field of neocortical stem cells are important for refining area pattern. Expressed in rostral-high to caudal-low gradients, the transcription factors Pax6 and Sp8 promote rostral area formation, whereas the transcription factor Emx2 is expressed in an opposing gradient and promotes a caudal identity. Of these transcription factors, evidence supports a patterning role for Sp8 and Emx2, whereas the effects of Pax6 on area patterning have been recently suggested by both gain- and lossof-function studies to be indirect (Fig. 3B) (Manuel et al. 2007; Pinon et al. 2008).

Sp8 is the vertebrate homolog of the Drosophila Buttonhead (Btd) gene and is a zinc factor transcription factor expressed in the developing nervous system. It is strongly expressed in the commissural ridge at E8/E8.5 and at E9.5 is expressed in a rostral-high to caudal-low gradient across the neocortex. The removal of Sp8 from the developing cortex results in a significant expansion of caudal areas at the expense of rostral areas, as assessed by cortical plate marker expression and corticalthalamic connectivity (Fig. 3B) (Zembrzycki et al. 2007). Sp8 is induced by FGF8 and can reciprocally regulate the expression of FGF8 (Sahara et al. 2007). Sp8 may act directly to promote a rostral identity in the neocortical stem cells, or indirectly by regulating the expression of Fgf8 in the commissural plate. Although Sp8 expression is also lost in the commissural plate of the cortex-specific knockout, no change in FGF8 expression in this region was detected, and this together with the graded expression of Sp8 in neocortical stem cells supports a direct role for this gene in the patterning of neocortical stem cells (Zembrzycki et al. 2007). Additionally, in vitro, Sp8 interacts on at a protein-protein level with the caudal transcription factor Emx2, and Emx2 can repress the induction of Fgf8 by Sp8, indicating that these two transcription factors might cross-regulate one another (Zembrzycki et al. 2007).

The vertebrate homolog of the Drosophila empty spiracles gene, Emx2, encodes a homeodomain protein that is expressed in the rostrolateral neural plate from E8.5 (Simeone et al. 1992; Gulisano et al. 1996; Mallamaci et al. 1998). In the neocortical primordium, Emx2 is expressed in a caudomedial high to rostrolateral low expression gradient that is, like the COUP-TFI expression gradient, sensitive to FGF signaling (Fig. 3). Examination of mice with absent, reduced, normal, and elevated 
levels of Emx2 have revealed that rostrocaudal area patterning shows a predictable and measured response to Emx2 dosage (Hamasaki et al. 2004). The loss of Emx2 results in a contraction of caudal areas and the expansion of rostral areas based on regionalized gene expression in the cortical plate, with heterozygous mice showing an intermediate phenotype (Fig. 3) (Bishop et al. 2002; Muzio et al. 2002). In contrast, in mice overexpressing Emx2 under the control of the Nestin promoter (ne-Emx2), there is an expansion of the caudal visual area 1 (V1) and contraction of rostral motor cortex (Hamasaki et al. 2004).

The shifts in area patterning observed in Emx2 mutant mice are smaller than those observed in COUP-TFI mutants, and the COUP-TFI expression gradient is unchanged in the neocortex of Emx2 null mice. These observations, together with a reduction in Emx2 expression observed in COUP-TFI null mice, indicate that Emx2 functions downstream of COUP-TFI to refine the position of area boundaries.

Like COUP-TFI, the expression of Emx2 is repressed by FGF8 signaling. The augmentation of the rostral FGF8 source by in vivo electroporation steepens the Emx2 gradient, whereas reduction of the endogenous FGF8 source, either by the electroporation of sFGFR3 or genetically in FGF8 hypomorphic mice, causes an increase in rostral Emx2 expression that flattens, and nearly abolishes, the Emx2 gradient (Fukuchi-Shimogori and Grove 2003).

In turn, Emx2 has been shown to negatively regulate FGF signaling. In the commissural plate of Emx2 mutants, the expression of Fgf8 and Fgf17 is up-regulated and Fgf15 is more generally up-regulated in the telencephalon (Cholfin and Rubenstein 2008). Conversely the endogenous expression of FGFs from the commissural plate can be repressed by the electroporation of an Emx2 expressing plasmid (Fukuchi-Shimogori and Grove 2003). These observations suggest that the patterning shifts observed in Emx2 null mice might be caused by elevated levels of FGF signaling, and this idea has been tested by reducing rostral FGF levels in Emx2 mutant animals. It was found that the shifts observed in the Emx2 null neocortex can be rescued by reducing endogenous FGF levels via electroporation of a soluble FGF receptor (Fukuchi-Shimogori and Grove 2003). Furthermore, the shifts can also be substantially rescued by crossing Emx2 null mice to a FGF17 null background (Cholfin and Rubenstein 2008). The observations that Emx2 can bind Sp8 and inhibit the Sp8 mediated induction of Fgf8 expression provide a potential mechanism by which the negative regulation of FGF signaling by Emx 2 may be accomplished (Sahara et al. 2007).

Therefore, FGF signaling also controls the graded expression of transcription factors other than COUP-TFI, most notably Sp8 and Emx2, across the developing neocortex, which function in turn to cross-regulate Fgf signaling and fine-tune the position of area boundaries (Fig. 4). It is likely that significant components of the neocortical patterning network remain to be discovered. In particular, it is probable that COUP-TFI regulates the expression of unknown factors that are important for caudal area identities.

\section{TRANSLATING TRANSCRIPTION FACTOR GRADIENTS TO AREA-SPECIFIC EXPRESSION PATTERNS}

Gradients function to pattern tissues by directing different cellular fates at different thresholds of the graded signal. During embryogenesis, developmental gradients are typically translated into distinct domains of gene expression. In the ventral spinal cord, stem cells are initially spatially patterned by gradients of gene expression in response to Shh signaling. In a dynamic and progressive process involving the use of cross-regulatory interactions, these gradients are then translated into molecularly distinct domains or pools of stem cells that are clearly delineated from one another (Dessaud et al. 2008). These domains of stem cells then give rise to spatially appropriate neuronal progeny (Fig. 5). Similarly, in the early Drosophila embryo, the gap genes interpret gradients to define spatially discrete populations of stem cells (Ephrussi and St. Johnston 2004). 
A

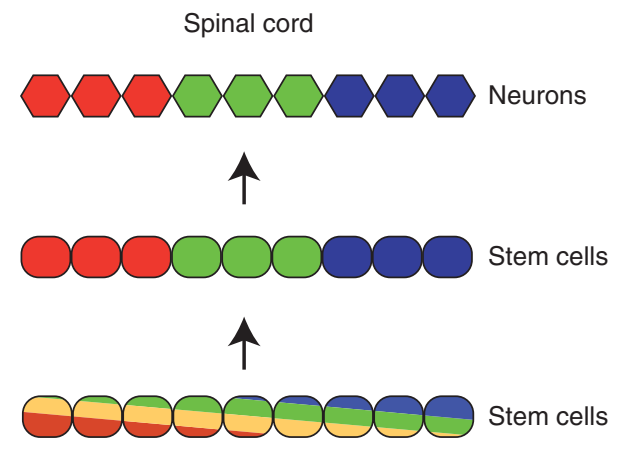

B

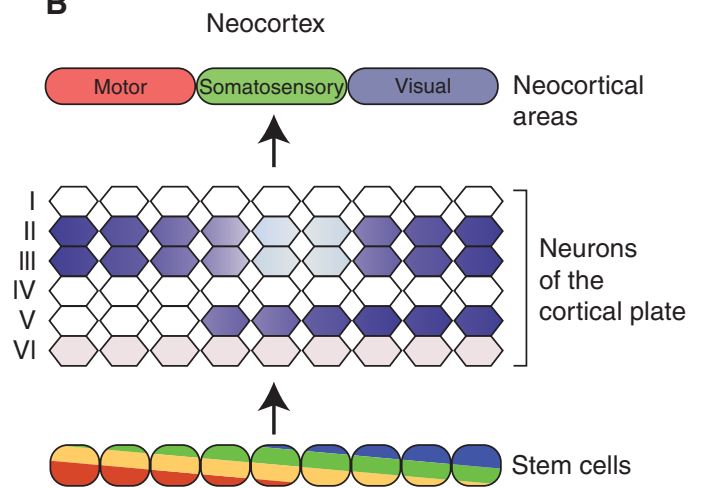

Figure 5. The translation of a gradient-based neocortical map in stem cells into spatially discrete patterns of neuronal gene expression in the neocortex. $(A)$ In the developing spinal cord, an initial graded pattern of transcription factor expression along the dorsoventral axis is progressively resolved into clearly delineated, molecularly distinct stem cell domains. Each spatially defined group of stem cells goes on to produce different classes of spinal cord neurons. (B) In contrast, in the developing neocortex, gradients of expression in the ventricular zone do not resolve into discrete spatial domains. Instead, those gradients appear to be translated into discrete spatial expression in the neurons generated by those stem cells. Furthermore, the spacial expression of specific genes often differs between different neuronal layers, as shown here for the gene Epha7.

In the cortical plate of the maturing neocortex, as discussed previously, complex domains of gene expression foreshadow the emergence of area boundaries, and it might be expected that these domains of gene expression are, in turn, prefigured by domains of expression in neocortical stem cells, in a situation comparable to the formation of stem pools across the dorsoventral axis in the spinal cord. However, extensive genomics screens for discrete rostrocaudal domains of expression in neocortical stem cells found no evidence for such domains, but rather revealed the graded expression of many more genes along this axis (Sansom et al. 2005). The role of these gradients in encoding spatial information is illustrated by experiments perturbing the high-caudal to low-rostral gradient of Emx2. On the measured increase or decrease of the Emx2 protein gradient, predictable rostral-caudal shifts in area position are observed (Hamasaki et al. 2004).

A key question then is how the gradients of gene expression in stem cells are read out to produce regionalized gene expression in the cortical plate. Recent studies on the transcription factor Bhlhb5 suggest that the gradients of expression in stem cells are transferred into graded expression of transcription factors in neurons along the rostrocaudal axis (Joshi et al. 2008). Bhlhb5 is initially expressed in a high caudomedial to low rostrolateral gradient in all cortical neurons of layers 2-5. Subsequently, this gradient retracts from the rostral cortex and is tuned into discrete domains of sharply bordered expression between the sensory and motor cortex as the cortical plate matures. In the absence of Bhlhb5, the molecular identity of somatosensory and caudal motor cortex is severely perturbed, indicating that this gene is crucial for specifying area fate (Joshi et al. 2008). Thus, a currently poorly understood patterning process occurs in postmitotic neurons to translate gradients of expression into discrete domains of gene expression that reflect the organization of cortical areas (Fig. 5B).

One striking feature of many of the known area-specific patterns of gene expression in neurons is the difference in spatial expression in different layers. For example, the Id 2 gene has markedly different spatial expression in layer-5 neurons compared with its expression in the neurons of layers 2 and 3 (Garel et al. 2003). Therefore, it is not the case that each stem cell has a spatial code that it passes on to all progeny equally. Rather, these observations 
suggest that neocortical stem cells interpret spatial information encoded by gradients of gene expression together with temporal information to generate the appropriate spatial pattern in each neocortical layer.

\section{THE CLONAL ANATOMY OF AREA SPECIFICATION}

Evidence is now emerging for a possible mechanism by which the graded spatial information present in neocortical stem cells may be precisely mapped onto the fundamental functional neuronal building blocks of the cortex, and thereby generates the columns and areas of the mature cortex. Within areas of the adult neocortex, neurons are typically arranged in columns that can be defined on the basis of functional, molecular, or connectivity properties, and typically consist of thousands of neurons. For example, in the primary visual cortex alone, ocular dominance, orientation, hyper and color columns have been identified (Hubel et al. 1977).

These functional columns can be further subdivided. Early investigators, using light microscopy and Nissl staining, discovered that cortical neurons are arranged in fine radial columns (De Felipe and Jones 1988). These radial columns, now often referred to as mini or microcolumns, consist of stereotypically vertically interconnected arrays of neurons that span the neocortical layers and share extrinsic connectivity (Jones 2000; Rakic 2008). Contrary to initial reports, microcolumns have been shown to vary in neuronal number, composition, and size. In the human temporal cortex, evidence has been found for repeating chains of about 11 neurons (Buldyrev et al. 2000), whereas the modular columns of pyramidal cells in the visual cortex of the monkey are typically comprised of 142 neurons (Peters and Yilmaz 1993). In an attractive hypothesis, microcolumns are viewed as the fundamental functional processing units of the neocortex underlying all broader divisions (Mountcastle 1998).

The studies that verified the radial unit hypothesis found that the neuronal progeny of neocortical stem cells form vertical arrays of neurons, and these have been described as the ontogenic units or columns of the neocortex (Rakic 1988a). Like functional microcolumns, these ontogenic columns, or radial clones, are not uniform but differ in structure between areas (Rakic 1988a). An attractive theory has been that the mini or microcolumns of highly connected neurons in the adult are groups of clonally related neurons. In support of this, it has recently been reported that radially aligned, clonally related neurons preferentially connect to one another, suggesting that ontogenic units/radial clones may be a component of functional microcolumns (Yu et al. 2009). If this observation generalizes to different parts of the cortex, it would provide a potentially powerful framework for integrating spatial patterning of the cortex with the formation of vertically arranged, area-specific circuits within cortical columns. Effectively, each stem cell would produce a vertical chain of highly connected neurons specific to a region of the cortex, and this simple functional unit would be the most basic component of a cortical column and area: The spatial identity of the stem cell would then be read out as the production of a region-appropriate unit of neurons.

\section{EVOLUTIONARY IMPLICATIONS OF A GRADIENT-BASED NEOCORTICAL PATTERNING SYSTEM}

The neocortex has rapidly evolved both in size and complexity during the evolution of the mammalian phyla. Although the simplest mammals have only 10-20 neocortical areas, modern studies indicate that humans possess as many as 100 distinct neocortical areas (Mountcastle 1998). However, across evolution, the mammalian neocortex retains the same basic areal organization, and it is clear that the area pattern is progressively elaborated over evolutionary time (Striedter 2005).

The gradient based neocortical patterning mechanism described here provides the means by which area pattern may have been conserved and elaborated as the neocortex increased in surface area and complexity. The expansion of 
neocortical surface area throughout evolution is thought to be because of an increase in the number of divisions undertaken by neocortical stem cells, which has in turn resulted in an increased number of ontogenic columns (Mountcastle 1998).

In line with this theory, a gradient-based patterning mechanism could readily scale as the number of ontogenic units or clones increased, facilitating the observed increase in cortical surface area while maintaining relative area sizes and positions. If clones do comprise some form of basic functional unit of the cortex, expanding stem cell number would simply increase the number of processing units available to form areas. Furthermore, the gradient-based patterning system described here, together with the gain of ontogenic units observed across evolution, provides a means for novel area acquisition. As the neocortex increased in size, new area identities along the rostro-caudal axis may have been acquired by the refinement of the mechanisms that interpret the gradients patterning neocortical stem cells, and/or by the elaboration of the gradient-based pattern in neocortical stem cells.

\section{CONCLUSIONS}

In the developing neocortex, area identity is specified by gradients of transcription factors established by morphogen gradient(s). An important feature of this system is that it does not operate in a linear manner but rather functions as a network (Fig. 4). At the core of the network, Fgf signaling establishes a gradient of the transcription factor COUP-TFI, a process modulated by the concomitant actions of Emx2, which acts to repress Fgf signaling and fine-tune area position.

Although this patterning system shares features with the patterning of other tissues by similar gradients, it also has several striking differences. In the spinal cord, a single morphogen, Shh, is responsible for patterning the ventral half of the dorsoventral axis. In contrast, in the neocortex, different varieties of the morphogen, including FGF8, FGF17, and FGF15, act together to specify rostrocaudal fate.
Intriguingly, evidence is now emerging that suggests FGF8 acts to induce the expression of FGF17 and FGF15 and that the different FGF ligands specify distinct aspects of the neocortical protomap. Recent evidence argues that FGF8 acts to repress COUP-TFI signaling, whereas FGF17 has a cross-regulatory interaction with Emx2 (Cholfin and Rubenstein 2008).

Similar to the situation in the spinal cord, Fgf signaling from the rostral pole of the neocortex sets up gradients of transcription factors by both activating and repressing gene expression. However, in contrast to the spinal cord and the early Drosophila embryo, these gradients are not then refined into distinct stem cell domains. In the neocortex, stem cells patterned by gradients give rise to spatially patterned progeny. Strikingly, neocortical stem cells give rise to differently spatially patterned neuronal progeny at different times. These observations suggest that neocortical stem cells interpret spatial information encoded by gradients of gene expression together with temporal information to generate the appropriate spatial pattern in each neocortical layer. Therefore, in a mechanism unique to the neocortex, graded information is resolved into region-specific gene expression in the postmitotic progeny of the stem cells.

\section{REFERENCES}

Anderson SA, Eisenstat DD, Shi L, Rubenstein JL. 1997. Interneuron migration from basal forebrain to neocortex: Dependence on Dlx genes. Science 278: 474-476.

Armentano M, Chou SJ, Tomassy GS, Leingartner A, O'Leary DD, Studer M. 2007. COUP-TFI regulates the balance of cortical patterning between frontal/motor and sensory areas. Nat Neurosci 10: 1277-1286.

Bachler M, Neubüser A. 2001. Expression of members of the Fgf family and their receptors during midfacial development. Mech Dev 100: 313-316.

Barbe MF, Levitt P. 1991. The early commitment of fetal neurons to the limbic cortex. J Neurosci 11: 519-533.

Bishop KM, Rubenstein JLR, O'Leary DDM. 2002. Distinct actions of Emx1, Emx2, and Pax6 in regulating the specification of areas in the developing neocortex. $J$ Neurosci 22: 7627-7638.

Briscoe J, Ericson J. 2001. Specification of neuronal fates in the ventral neural tube. Curr Opin Neurobiol 11: $43-49$. 
Brodmann K. 2006. Brodmann's localisation in the cerebral cortex. Springer, New York.

Buchwalter G, Gross C, Wasylyk B. 2004. Ets ternary complex transcription factors. Gene 324: 1-14.

Buldyrev SV, Cruz L, Gomez-Isla T, Gomez-Tortosa E, Havlin S, Le R, Stanley HE, Urbanc B, Hyman BT. 2000. Description of microcolumnar ensembles in association cortex and their disruption in Alzheimer and Lewy body dementias. Proc Natl Acad Sci 97: 5039-5043.

Cholfin JA, Rubenstein JL. 2008. Frontal cortex subdivision patterning is coordinately regulated by Fgf8, Fgf17, and Emx2. J Comp Neurol 509: 144-155.

Cohen-Tannoudji M, Babinet C, Wassef M. 1994. Early determination of a mouse somatosensory cortex marker. Nature 368: 460-463.

Creutzfeldt OD. 1977. Generality of the functional structure of the neocortex. Naturwissenschaften 64: 507-517.

De Felipe J, Jones EG. 1988. Cajal on the cerbral cortex. In An annotated translation of the complete writings. Oxford University Press, New York.

de Launoit Y, Baert JL, Chotteau-Lelievre A, Monte D, Coutte L, Mauen S, Firlej V, Degerny C, Verreman K. 2006. The Ets transcription factors of the PEA3 group: Transcriptional regulators in metastasis. Biochim Biophys Acta 1766: 79-87.

Dessaud E, McMahon AP, Briscoe J. 2008. Pattern formation in the vertebrate neural tube: A sonic hedgehog morphogen-regulated transcriptional network. Development 135: 2489-2503.

Ephrussi A, St. Johnston D. 2004. Seeing is believing: The bicoid morphogen gradient matures. Cell 116: 143-152.

Ericson J, Briscoe J, Rashbass P, van Heyningen V, Jessell TM. 1997. Graded sonic hedgehog signaling and the specification of cell fate in the ventral neural tube. Cold Spring Harb Symp Quant Biol 62: 451-466.

Faedo A, Tomassy GS, Ruan Y, Teichmann H, Krauss S, Pleasure SJ, Tsai SY, Tsai MJ, Studer M, Rubenstein JL. 2008. COUP-TFI coordinates cortical patterning, neurogenesis, and laminar fate and modulates MAPK/ERK, AKT, and beta-catenin signaling. Cereb Cortex 18: 2117-2131.

Frost DO, Schneider GE. 1979. Plasticity of retinofugal projections after partial lesions of the retina in newborn Syrian hamsters. J Comp Neurol 185: 517-567.

Fukuchi-Shimogori T, Grove EA. 2001. Neocortex patterning by the secreted signaling molecule FGF8. Science 294: $1071-1074$

Fukuchi-Shimogori T, Grove EA. 2003. Emx2 patterns the neocortex by regulating FGF positional signaling. Nat Neurosci 6: 825-831.

Gaillard A, Nasarre C, Roger M. 2003. Early (E12) cortical progenitors can change their fate upon heterotopic transplantation. Eur J Neurosci 17: 1375-1383.

Garel S, Huffman KJ, Rubenstein JLR. 2003. Molecular regionalization of the neocortex is disrupted in Fgf8 hypomorphic mutants. Development 130: 1903-1914.

Gaspard N, Bouschet T, Hourez R, Dimidschstein J, Naeije G, van den Ameele J, Espuny-Camacho I, Herpoel A, Passante L, Schiffmann SN, et al. 2008. An intrinsic mechanism of corticogenesis from embryonic stem cells. Nature 455: 351-357.

Gitton Y, Cohen-Tannoudji M, Wassef M. 1999a. Role of thalamic axons in the expression of $\mathrm{H}-2 \mathrm{Z} 1$, a mouse somatosensory cortex specific marker. Cereb Cortex 9: 611-620.

Gitton Y, Cohen-Tannoudji M, Wassef M. 1999b. Specification of somatosensory area identity in cortical explants. J Neurosci 19: 4889-4898.

Gulisano M, Broccoli V, Pardini C, Boncinelli E. 1996. Emx1 and Emx2 show different patterns of expression during proliferation and differentiation of the developing cerebral cortex in the mouse. Eur J Neurosci 8: 1037-1050.

Hamasaki T, Leingärtner A, Ringstedt T, O'Leary DDM. 2004. EMX2 regulates sizes and positioning of the primary sensory and motor areas in neocortex by direct specification of cortical progenitors. Neuron 43: 359-372.

Hébert JM, Lin M, Partanen J, Rossant J, McConnell SK. 2003. FGF signaling through FGFR1 is required for olfactory bulb morphogenesis. Development 130: 11011111.

Hubel DH, Wiesel TN, Stryker MP. 1977. Orientation columns in macaque monkey visual cortex demonstrated by the 2-deoxyglucose autoradiographic technique. Nature 269: 328-330.

Jones EG. 2000. Microcolumns in the cerebral cortex. Proc Natl Acad Sci 97: 5019-5021.

Joshi PS, Molyneaux BJ, Feng L, Xie X, Macklis JD, Gan L. 2008. Bhlhb5 regulates the postmitotic acquisition of area identities in layers II-V of the developing neocortex. Neuron 60: 258-272.

Kornack DR, Rakic P. 1995. Radial and horizontal deployment of clonally related cells in the primate neocortex: Relationship to distinct mitotic lineages. Neuron 15: 311-321.

Kuljis RO, Rakic P. 1990. Hypercolumns in primate visual cortex can develop in the absence of cues from photoreceptors. Proc Natl Acad Sci 87: 5303-5306.

Lein ES, Hawrylycz MJ, Ao N, Ayres M, Bensinger A, Bernard A, Boe AF, Boguski MS, Brockway KS, Byrnes EJ, et al. 2007. Genome-wide atlas of gene expression in the adult mouse brain. Nature 445: 168-176.

Liu Q, Dwyer ND, O'Leary DD. 2000. Differential expression of COUP-TFI, CHL1, and two novel genes in developing neocortex identified by differential display PCR. J Neurosci 20: 7682-7690.

Loncar D, Singer SJ. 1995. Cell membrane formation during the cellularization of the syncytial blastoderm of Drosophila. Proc Natl Acad Sci 92: 2199-2203.

Mackarehtschian K, Lau CK, Caras I, McConnell SK. 1999. Regional differences in the developing cerebral cortex revealed by ephrin-A5 expression. Cereb Cortex 9: 601-610.

Mallamaci A, Iannone R, Briata P, Pintonello L, Mercurio S, Boncinelli E, Corte G. 1998. EMX2 protein in the developing mouse brain and olfactory area. Mech Dev 77: 165-172.

Manuel M, Georgala PA, Carr CB, Chanas S, Kleinjan DA, Martynoga B, Mason JO, Molinek M, Pinson J, 
Pratt T, et al. 2007. Controlled overexpression of Pax6 in vivo negatively autoregulates the Pax6 locus, causing cell-autonomous defects of late cortical progenitor proliferation with little effect on cortical arealization. Development 134: 545-555.

Mason JM, Morrison DJ, Basson MA, Licht JD. 2006. Sprouty proteins: Multifaceted negative-feedback regulators of receptor tyrosine kinase signaling. Trends Cell Biol 16: $45-54$.

McConnell SK. 1988. Fates of visual cortical neurons in the ferret after isochronic and heterochronic transplantation. J Neurosci 8: 945-974.

Minowada G, Jarvis LA, Chi CL, Neubüser A, Sun X, Hacohen N, Krasnow MA, Martin GR. 1999. Vertebrate Sprouty genes are induced by FGF signaling and can cause chondrodysplasia when overexpressed. Development 126: 4465-4475.

Miyashita-Lin EM, Hevner R, Wassarman KM, Martinez S, Rubenstein JL. 1999. Early neocortical regionalization in the absence of thalamic innervation. Science 285: 906-909.

Mountcastle VB. 1998. The cerebral cortex. Harvard University Press, Cambridge, Massachusetts.

Muzio L, DiBenedetto B, Stoykova A, Boncinelli E, Gruss P, Mallamaci A. 2002. Emx2 and Pax6 control regionalization of the pre-neuronogenic cortical primordium Cereb Cortex 12: 129-139.

Nakagawa Y, Johnson JE, O’Leary DD. 1999. Graded and areal expression patterns of regulatory genes and cadherins in embryonic neocortex independent of thalamocortical input. J Neurosci 19: 10877-10885.

Nothias F, Fishell G, Ruiz i Altaba A. 1998. Cooperation of intrinsic and extrinsic signals in the elaboration of regional identity in the posterior cerebral cortex. Curr Biol 8: 459-462.

O'Leary DD. 1989. Do cortical areas emerge from a protocortex? Trends Neurosci 12: 400-406.

O'Rourke NA, Sullivan DP, Kaznowski CE, Jacobs AA, McConnell SK. 1995. Tangential migration of neurons in the developing cerebral cortex. Development 121: 2165-2176.

Peters A, Yilmaz E. 1993. Neuronal organization in area 17 of cat visual cortex. Cereb Cortex 3: 49-68.

Pinon MC, Tuoc TC, Ashery-Padan R, Molnar Z, Stoykova A. 2008. Altered molecular regionalization and normal thalamocortical connections in cortex-specific Pax6 knock-out mice. J Neurosci 28: 8724-8734.

Price J, Thurlow L. 1988. Cell lineage in the rat cerebral cortex: A study using retroviral-mediated gene transfer. Development 104: 473-482.

Rakic P. 1988a. Specification of cerebral cortical areas Science 241: 170-176.

Rakic P. 1990. Radial unit hypothesis of cerebral cortical evolution. In Principles and design and operation of the brain (ed. J.C. Eccles and O. Creutzfeldt), pp. 25-48. Vatican City: Pontificae Academiae Scripta Varia.

Rakic P. 1991. Experimental manipulation of cerebral cortical areas in primates. Philos Trans $R$ Soc Lond $B$ Biol Sci 331: 291-294.

Rakic P. 2008. Confusing cortical columns. Proc Natl Acad Sci 105: $12099-12100$
Rakic PSW. 1988b. Intrinsic and extrinsic determinants of neocortical parcelation: A radial unit model. In Neurobiology of the neocortex, pp. 2-28. New York, Wiley.

Rubenstein JL, Anderson S, Shi L, Miyashita-Lin E, Bulfone A, Hevner R. 1999. Genetic control of cortical regionalization and connectivity. Cereb Cortex 9: 524-532.

Sahara S, Kawakami Y, Izpisua Belmonte JC, O'Leary DD. 2007. Sp8 exhibits reciprocal induction with Fgf8 but has an opposing effect on anterior-posterior cortical area patterning. Neural Develop 2: 10.

Salas R, Petit FG, Pipaon C, Tsai MJ, Tsai SY. 2002. Induction of chicken ovalbumin upstream promotertranscription factor I (COUP-TFI) gene expression is mediated by ETS factor binding sites. Eur J Biochem 269: 317-325.

Sansom SN, Hébert JM, Thammongkol U, Smith J, Nisbet G, Surani MA, McConnell SK, Livesey FJ. 2005. Genomic characterisation of a Fgf-regulated gradientbased neocortical protomap. Development 132: $3947-$ 3961.

Sato T, Joyner AL, Nakamura H. 2004. How does Fgf signaling from the isthmic organizer induce midbrain and cerebellum development? Dev Growth Differ 46: 487-494.

Schlaggar BL, O’Leary DD. 1991. Potential of visual cortex to develop an array of functional units unique to somatosensory cortex. Science 252: 1556-1560.

Simeone A, Gulisano M, Acampora D, Stornaiuolo A, Rambaldi M, Boncinelli E. 1992. Two vertebrate homeobox genes related to the Drosophila empty spiracles gene are expressed in the embryonic cerebral cortex. Embo J11: 2541-2550.

Soriano E, Dumesnil N, Auladell C, Cohen-Tannoudji M, Sotelo C. 1995. Molecular heterogeneity of progenitors and radial migration in the developing cerebral cortex revealed by transgene expression. PNAS 92: $11676-$ 11680.

Stoykova A, Gruss P. 1994. Roles of Pax-genes in developing and adult brain as suggested by expression patterns. J Neurosci 14: 1395-1412.

Striedter G. 2005. Principles of brain evolution. Sinauer, Sunderland, Massachusetts.

Sur M, Garraghty PE, Roe AW. 1988. Experimentally induced visual projections into auditory thalamus and cortex. Science 242: 1437-1441.

Tan SS, Breen S. 1993. Radial mosaicism and tangential cell dispersion both contribute to mouse neocortical development. Nature 362: 638-640.

Thomson AM, Lamy C. 2007. Functional maps of neocortical local circuitry. Front Neurosci 1: 19-42.

van der Loos H, Woolsey TA. 1973. Somatosensory cortex: Structural alterations following early injury to sense organs. Science 179: 395-398.

Walsh C, Cepko CL. 1992. Widespread dispersion of neuronal clones across functional regions of the cerebral cortex. Science 255: 434-440.

Walsh C, Cepko CL. 1993. Clonal dispersion in proliferative layers of developing cerebral cortex. Nature 362: $632-635$. 


\section{S.N. Sansom and F.J. Livesey}

Walther C, Gruss P. 1991. Pax-6, a murine paired box gene, is expressed in the developing CNS. Development 113: 1435-1449.

Wonders CP, Anderson SA. 2006. The origin and specification of cortical interneurons. Nat Rev Neurosci 7: 687-696.

Wree A, Zilles K, Schleicher A. 1983. A quantitative approach to cytoarchitectonics. VIII. The areal pattern of the cortex of the albino mouse. Anat Embryol (Berl) 166: $333-353$.
Yu YC, Bultje RS, Wang X, Shi SH. 2009. Specific synapses develop preferentially among sister excitatory neurons in the neocortex. Nature 458: 501-504.

Zembrzycki A, Griesel G, Stoykova A, Mansouri A. 2007. Genetic interplay between the transcription factors Sp8 and Emx2 in the patterning of the forebrain. Neural Develop 2: 8 .

Zhou C, Tsai SY, Tsai MJ. 2001. COUP-TFI: An intrinsic factor for early regionalization of the neocortex. Genes and Dev 15: 2054-2059. 


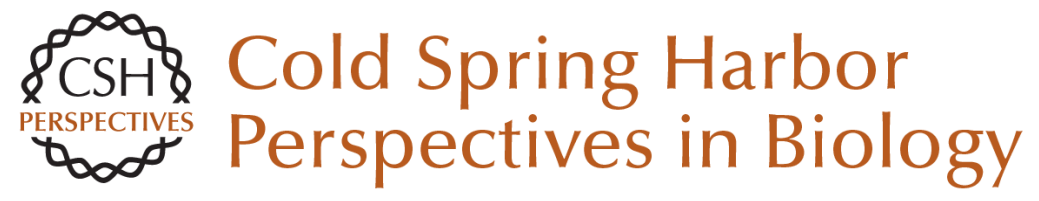

\section{Gradients in the Brain: The Control of the Development of Form and Function in the Cerebral Cortex}

Stephen N. Sansom and Frederick J. Livesey

Cold Spring Harb Perspect Biol 2009; doi: 10.1101/cshperspect.a002519

Subject Collection Generation and Interpretation of Morphogen Gradients

Regulation of Organ Growth by Morphogen

Gradients

Gerald Schwank and Konrad Basler

Signaling Gradients during Paraxial Mesoderm

Development

Alexander Aulehla and Olivier Pourquié

Morphogen Gradient Formation

Ortrud Wartlick, Anna Kicheva and Marcos

González-Gaitán

Nodal Morphogens

Alexander F. Schier

Gradients and the Specification of Planar Polarity in the Insect Cuticle

David Strutt

Vertebrate Limb Development: Moving from

Classical Morphogen Gradients to an Integrated

4-Dimensional Patterning System

Jean-Denis Bénazet and Rolf Zeller

Establishing and Interpreting Graded Sonic

Hedgehog Signaling during Vertebrate Neural

Tube Patterning: The Role of Negative Feedback

Vanessa Ribes and James Briscoe
Gradients in Planarian Regeneration and

Homeostasis

Teresa Adell, Francesc Cebrià and Emili Saló

Shaping Morphogen Gradients by Proteoglycans

Dong Yan and Xinhua Lin

Forming Patterns in Development without

Morphogen Gradients: Scattered Differentiation

and Sorting Out

Robert R. Kay and Christopher R.L. Thompson

Robust Generation and Decoding of Morphogen

Gradients

Naama Barkai and Ben-Zion Shilo

Models for the Generation and Interpretation of

Gradients

Hans Meinhardt

Graded Dorsal and Differential Gene Regulation in

the Drosophila Embryo

Gregory T. Reeves and Angelike Stathopoulos

Chemical Gradients and Chemotropism in Yeast Robert A. Arkowitz

For additional articles in this collection, see http://cshperspectives.cshlp.org/cgi/collection/

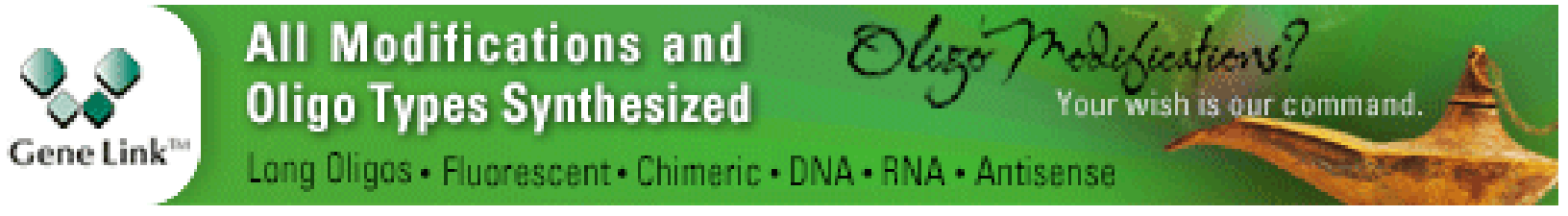

Copyright @ 2009 Cold Spring Harbor Laboratory Press; all rights reserved 
Systems Biology of the Self-regulating

Morphogenetic Gradient of the Xenopus Gastrula Jean-Louis Plouhinec and E. M. De Robertis
Gradients in the Brain: The Control of the Development of Form and Function in the Cerebral Cortex

Stephen N. Sansom and Frederick J. Livesey

For additional articles in this collection, see http://cshperspectives.cshlp.org/cgi/collection/

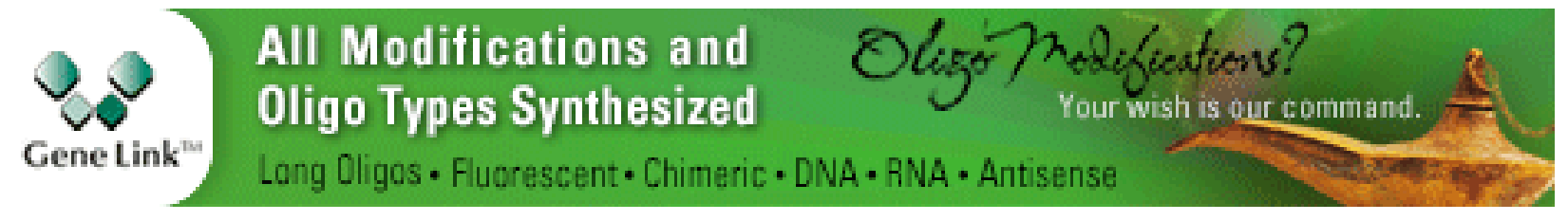

Copyright @ 2009 Cold Spring Harbor Laboratory Press; all rights reserved 